\title{
Adaptive Clustering for Device Free User Positioning utilizing Passive RFID
}

\section{Benjamin Wagner}

University of Rostock

Institute of Applied

Microelectronics and CE

Rostock, Germany

benjamin.wagner@uni-rostock.de

\section{Dirk Timmermann}

University of Rostock

Institute of Applied

Microelectronics and CE

Rostock, Germany

dirk.timmermann@uni-rostock.de
Permission to make digital or hard copies of all or part of this work for personal or classroom use is granted without fee provided that copies are not made or distributed for profit or commercial advantage and that copies bear this notice and the full citation on the first page. To copy otherwise, or republish, to post on servers or to redistribute to lists, requires prior specific permission and/or a fee. Request permissions from permissions@acm.org.

UbiComp'13 Adjunct, Sept 8-12, 2013, Zurich, Switzerland.

ACM 978-1-4503-2139-6/13/09...\$15.00.

http://dx.doi.org/10.1145/2494091.2497608

\begin{abstract}
Context sensing is an important part of building

ubiquitous smart and assistive environments. It is the

major data source for intention recognition and

strategy generation systems. Device-free localization systems (DFL) join the efforts of non-instrumentation

of users maintaining their privacy.

In recent publications we propose an innovative approach utilizing a cluster of passive Radio Frequency Identification Transponders (pRFID) for device-free radio-based positioning. Due to the point that the RFID technology is typically not designed for that purpose we have to deal with certain drawbacks. A high number of transponders typically conclude in lower measurement frame rates while generating substantially more information for accurate positioning.
\end{abstract}

To fix this tradeoff this work presents a transponder clustering approach based on inherent EPC protocol based bit masking, which allows us to calculate fast coarse grained localization results and increase the precision by time, so that the user is able to adjust between localization speed and accuracy.

We made simulations and conducted experiments in an indoor room DFL scenario for validation. 


\section{Author Keywords}

DFL, RFID, Received Signal Strength, RSSI, Smart Environments, Positioning, Wireless.

\section{ACM Classification Keywords}

Wireless communication, Data communications, Applications, Design studies

\section{Introduction}

Developing ubiquitous, assistive and self-organizing environments is mainly based on positioning information of the present users. The ubiquitous character defines the need for non-invasive, wireless, privacy preserving technologies. Device-free localization approaches provide these advantages with no need for user-attached radio hardware.

One approach is proposed by Lieckfeldt and Wagner [1], [2] replacing most of the active radio beacons with completely passive Radio Frequency Identification (pRFID) transponders. That combines the advantages of energy efficiency, because the transponders do not need batteries, and very easy deployment. They can be placed i.e. under the carpet or the wallpaper.

Furthermore pRFID transponders can be purchased very cheap, as low as $0.20 €$ per item. In the past multiple localization algorithms were proposed [2-6] providing positioning results with an error as low as 0.3 $\mathrm{m}$.

Since the RFID technology is typically used and designed as replacement for barcodes in the logistics area, entry controllers or theft protection, protocol and communication is optimized for simple tag recognition instead of link quality, signal strength or reading speed. Having a closer look on the pRFID approach reveals a strong trade off. High localization accuracy needs a high amount of sensor data, which can only be gathered with a high amount of time. Therefore a clustering algorithm is proposed in this paper providing fast continuous position estimation with increasing precision.

The Paper is structured as follows: after introducing important approaches the mentioned RFID tradeoff is illustrated in detail. In the third section the clustering approach is explained, followed by the experimental validation. In the last chapter we draw conclusions.

\section{Related Work}

\section{Model based Positioning}

The model of Lieckfeldt et al. [1], [3] can be substantially used to describe the influence of human presence on simple passive RFID communication between reader and transponders. The authors associate the measured Received Signal Strength Indicator (RSSI) provided by the reader hardware with the travelling path difference $d_{\text {exc }}$ between the LOS and NLOS path of the radio signal divided by the position of the scattering user. The proposed model can be mathematically described as:

$$
\Delta P\left(d_{\text {exc }}\right)=A d_{\text {exc }}^{B} \cos \left(\frac{2 \pi}{\lambda} d_{e x c}+\phi_{\text {refl }}\right)
$$

with $\triangle \mathrm{P}$ as estimated RSSI change, wave length $\lambda$ and phase shift $\phi_{\text {refl }}$ and is illustrated in Figure 1 . The parameter bundle $A, B, \lambda$ and $\phi_{\text {refl }}$ is subject to the experimental environment due to multipath fading effects. Therefore the model needs to be re-adjusted to each experimental setup. Due to the RFID protocol it is difficult to set a stable power value for every transponder. Therefore a 2 phase measurement was 
conducted: a calibration phase with no user presence and a measurement step with scatterer in the field.

$$
\Delta P=P_{\text {meas }}-P_{\text {cal }}
$$

with signal strength $P$ and RSSI difference vector $\Delta P$.

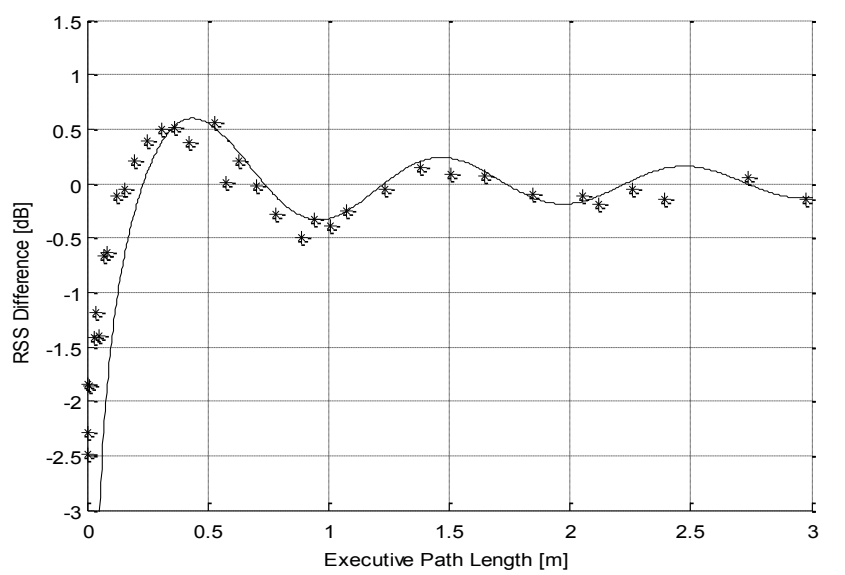

Figure 1. Theoretical model regression and experimental data points from multiple transponder scenario

Based on this model different methods for the localization of users were investigated:

Database based localization by minimizing a loglikelihood-function from the difference between the expected change of signal strength (due to the mentioned model) and the measurement. The results provide a maximum RMSE of $0.75 \mathrm{~m}$ at $95 \%$ confidence level [3], but the computation needs significant computational power due to complicated calculations. Thus it is not useable for highly accurate online localization.
Geometric localization based on Linear Least Squares and Intersection Points applied on the measured signal strength differences. The results provide lower accuracy at approximately $1.61 \mathrm{~m}$ [3], while having a lower time complexity.

For intention recognition purposes in smart environments an appropriate approach needs to combine high speed and accuracy.

\section{Imaging based Localization}

In our recent works [2], [4] wireless sensor network based radio tomographic imaging [7], [8] and pRFID DFL were combined. The setup consists of waist-high mounted passive transponders placed around the discretised measurement area. The RFID antennas are placed directly behind the transponder lines to guarantee a maximum power transfer.

The imaging result is calculated by using the model of Wilson et.al.[8]:

$$
\Delta y=W \Delta x+n
$$

with $\Delta y$ as matrix of RSS differences in $\mathrm{dB}, W$ as precalculated weighting matrix for every pixel-linkcombination, $n$ as zero mean gaussian noise vector and $\Delta x$ as matrix of pixel attenuations.

In Figure 2 sample images of the algorithm are illustrated, the center of the maximum pixel value is regarded as the most probable user location. The algorithm can locate human with as low as $0.3 \mathrm{~m}$ mean location error. In [2] we propose multiple improvements for performance and online operation. 

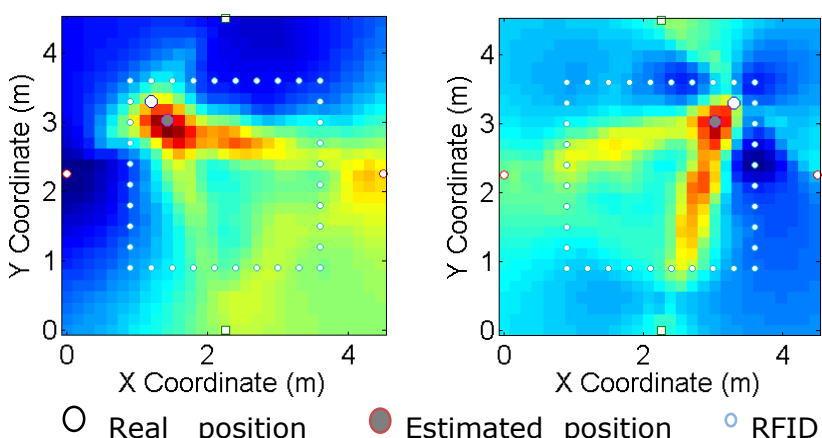

Figure 2. Passive RFID Tomographic Images

\section{Training based Localization}

There is much work done in the field of device based localization utilizing training approaches, e.g. Multilayered Perceptrons (MLP). Approaches are based on indoor Wi-Fi [9], Ultra-Wideband (UWB) [10] or Wireless Sensor Networks (WSN) [11]. In [5], [6] we propose a MLP-based device-free localization approach utilizing a passive RFID field. Therefore we implemented a three layered MLP getting the RSSI differences into its input layer and providing a 2D user position out of the output layer.

Evaluating different backpropagation training functions for training the neuron weighting and bias values and layered transfer functions it is possible to achieve accuracies as low as $0.01 \mathrm{~m}$ MSE in a ground mounted pRFID scenario.

\section{RFID Tradeoff}

The EPCglobal[12] Radio-Frequency Identity Protocol for Class-1 Generation-2 UHF RFID communication at $860-960 \mathrm{MHz}$ defines the tag-interrogator principles for so-called "Dense Reader Environments" in scenarios where multiple RFID readers (or interrogators) are working in the same environment. For scenarios were multiple transponders should be read the standard defines an inventory protocol based on slotted ALOHA collision avoidance. It is optimized for reading all transponders within the communication area.

Typically this number is not known, but in the mentioned approach this information is available a priori. In contrast to a logistic RFID scenario, a pRFID field in this approach consists of a high number of transponders, since the forward link between reader antenna and transponder is only regarded as power supply, and a relatively low number of receivers (RFID readers):

$$
n_{t x} \gg n_{r x}
$$

Key value of the mentioned approach is the number of measured transponder RSSI values in the experimental environment. To describe the relationship between different experimental setups and this value we introduce the density coefficient $C_{\text {dens }}$ as quotient between the surrounding transponder number (assuming a uniform tag distribution):

$$
C_{\text {dens }}=\frac{n_{t}}{A_{S}}=S_{P} \frac{n_{t}}{n_{P}}=S_{l} \frac{n_{t}}{\rho_{l}}
$$

with tag number $n_{t}$ and measurement field size $A_{S}$ (calculated due to the specific measurement geometry), number of Pixels $n_{P}$, link density $\rho_{l}$ and the correspondent scaling factors $S_{x}$. 


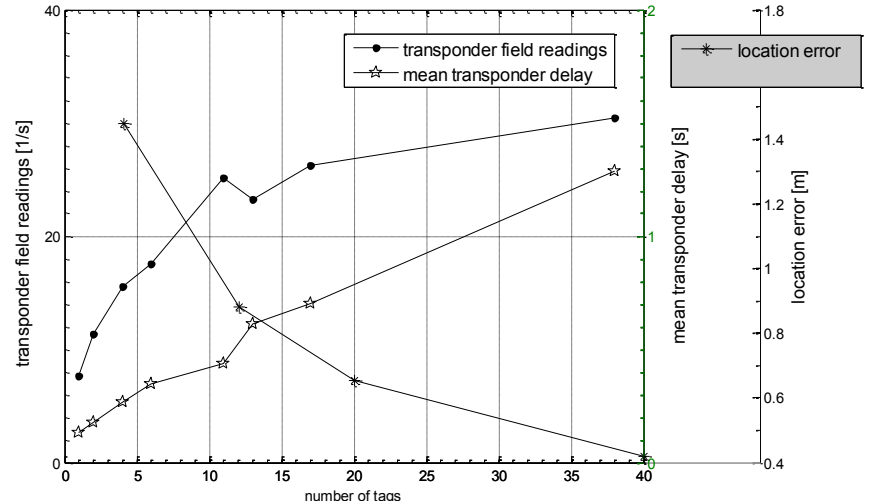

Figure 3. Mean readings, mean delays and mean location error for changing transponder numbers

Due to first simulations (cp. Figure 3 ) it is possible to state the two mentioned coherences between tag number and readings per second:

$$
\varepsilon_{l}=\mathrm{A} * \log \left(n_{t}\right)+\sigma_{r}
$$

and tag number and localization error:

$$
v_{l}=\mathrm{B} * \exp \left(-C * n_{t}\right)+\sigma_{e}
$$

\begin{tabular}{|c|c|}
\hline \multicolumn{2}{|l|}{ Parameters } \\
\hline $\mathrm{A}$ & 6.5890 \\
\hline$\sigma_{r}$ & 7.0440 \\
\hline$B$ & 1.5230 \\
\hline$C$ & 0.0909 \\
\hline$\sigma_{e}$ & 0.3873 \\
\hline
\end{tabular}

Table 1. Fitted model parameters
The parameter set $\{A, \sigma\}$ and $\{C, D, \sigma\}$ need to be determined for every new experimental setup. They define the place of a possible optimum between speed and accuracy. For our mentioned scenario of this paper we calculated the parameters due to Table 1 .

\section{Clustering Approach}

For getting a dynamic localization result we propose a 2-phase clustering approach. First we prior a high localization speed before getting a high accuracy result. Therefore we state a minimal Initialization Transponder Cluster $\bar{T}=\left\{t_{1} ; . . ; t_{x}\right\}$ with a minimal symmetric transponder matrix.

Table 2 is showing the clustering procedure in pseudo code. In phase 1 a first location estimate is calculated as a coarse grained result with high error. Due to this first position an area with a high certainty of user presence can be stated. Du to that area a tag cluster can be calculated due to the following equation:

$$
d_{i j}=\left\{\begin{array}{c}
1 \text { if } d_{t(i) p(j)}+d_{p(j) r x(i)}<d_{t(i) r x(i)}+\lambda \\
0 \quad \text { otherwise }
\end{array}\right.
$$

with $d_{i j}$ stating the dependency of link $i$ to position estimate $j$ generating a resulting matrix:

$$
\bar{D}_{s}=\operatorname{dim}\{\text { links, discrete user positions }\}
$$

The elliptic width $\lambda$ is directly adjusting the number of transponders connected to the individual link. $p(j)$ is denoting the users position, $t(i)$ and $r x(i)$ are denoting the corresponding transponder and reader antenna. Figure 4 is showing this relationship. The upper Bound for the elliptic curve is the maximum Fresnel zone for the specific communication link. 


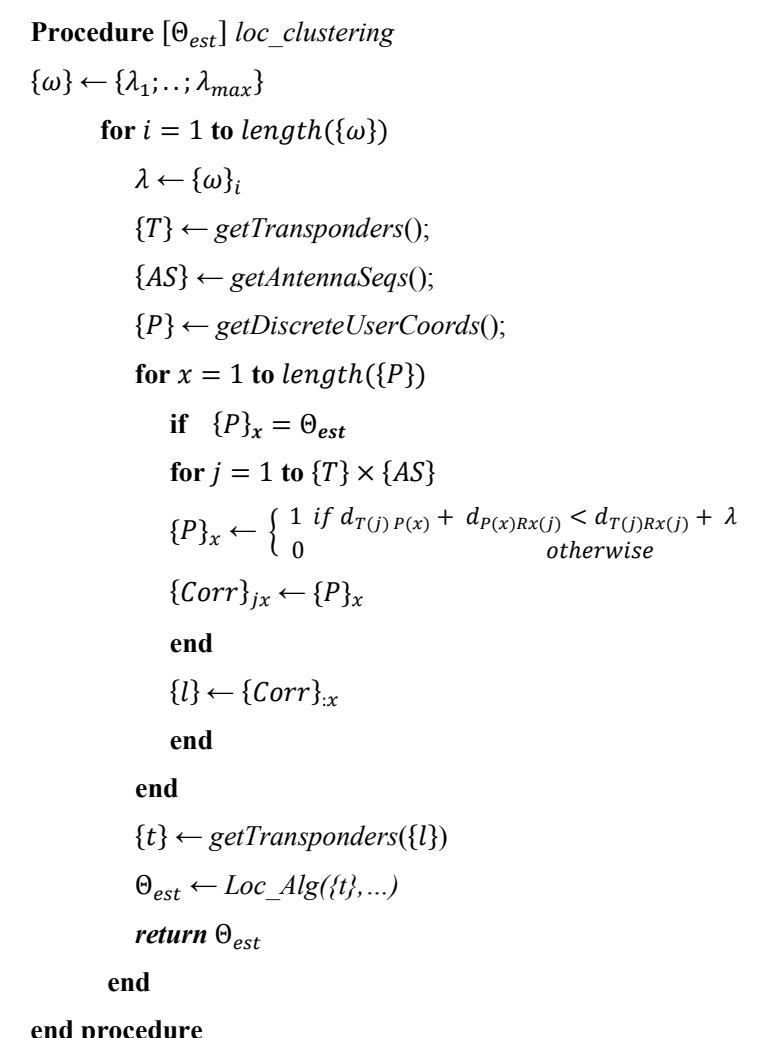

Table 2. Adaptive clustering algorithm

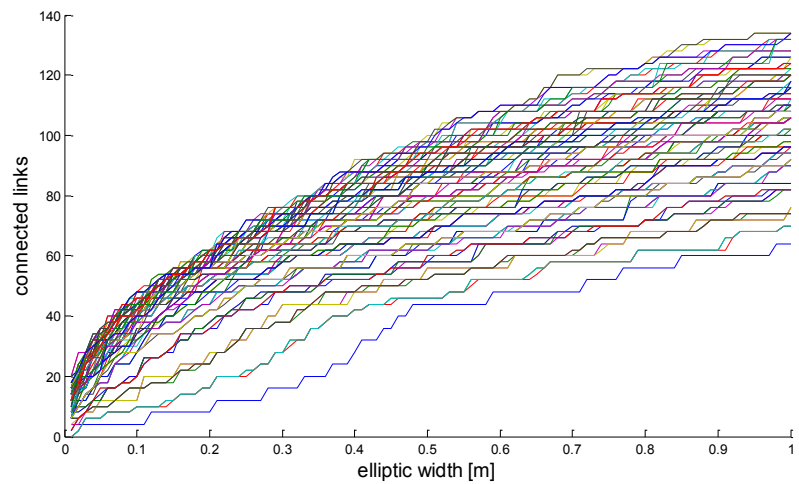

Figure 4. Cluster size with rising $\lambda$ factor for different initial position estimates

Due to the mentioned tradeoff between localization speed and error, we define a continuous localization process with decreasing localization error by time. Thus the user is able to get reasonable results after appropriate time period. In phase 2 the transponder clustering algorithm is started with a starting and a maximum cluster set. The algorithm stops at this maximum with the highest available position information.

\section{Experimental Validation}

The experimental setup is illustrated in Figure 5. It consists of three major parts: a passive UHF RFID system, a network layer and the processing workstation. We use a bistatic UHF reader from Alien Technology $®[13]$ working in the ISM $868 \mathrm{MHz}$

frequency band. For transponder powering and the backward link communication four linear polarized UHF antennas with a gain of $6 \mathrm{dBiL}$ and a 70 degree azimuth beamwidth are connected to its ports. For 
measurements we installed a square field (edge length: $3.5 \mathrm{~m}$ ) of hip height mounted UHF transponders with a 96-bit EPC[12] compliant memory holding a unique identification number.

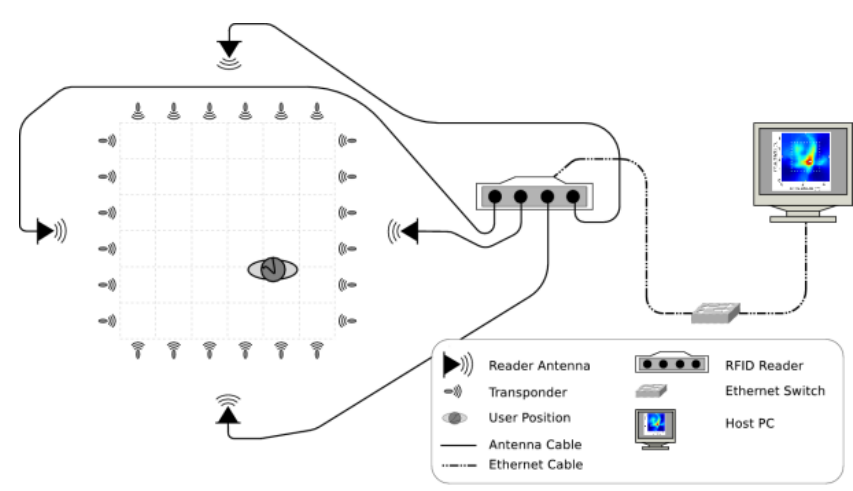

Figure 5. Experimental setup and system structure

The RFID system is connected via ethernet to the operating workstation. Every transponder answer is repeated via TCP packets to the workstation for further evaluation. The processing station is an Unix running PC with an Intel ${ }^{\circledR}$ Core $^{\mathrm{TM}} 2$ Quad CPU @ 4*3GHz.

The EPCglobal[12] Radio-Frequency Identity Protocol for Class-1 Generation-2 UHF RFID communication at 860-960 MHz defines baseband operations to address a smaller subset of RFID transponders. Therefore bit masking instructions are available in the readers API. Typically we have changing transponder group members due to the current position estimation. Therefore the individual 96-Bit EPC key is divided into hexadecimal subgroups used for group division.
As reference localization algorithm we used the pRFID tomography approach from Wagner et Patwari [2][4]. The evaluation script is -an integrated Java/Matlab $₫$ Script containing the RFID communication structure in Java and the evaluation code in the Matlab part.

In Figure 6 the initialization time results with raising transponder number is illustrated. As you can see the time for algorithm initialization is constant with rising clustering steps. Random peaks could be explained by the priority thread handling of the operating system. Within the initialization phase the parameters and matrices for location estimation are initialized.

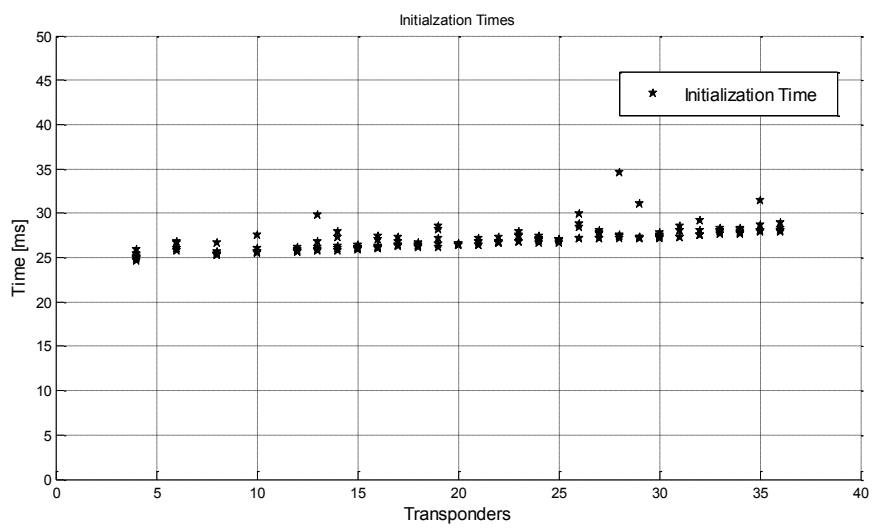

Figure 6. Localization algorithm initialization time

Strongly dependent on rising tag clusters is the location estimation time displayed in Figure 7. It is a nearly linear process because the computation is mainly based on matrix operations, which are determined by link and transponder number. A high number of radio links affects the results matrices in a linear way. As you can see taking the median value is a good choice to suppress peaks in the measurement data. 
Implementing the approach explained in chapter 4 leads to reasonable localization results reaching a maximum accuracy of $\sim 0.3 \mathrm{~m}$. After $\sim 1 \mathrm{~s}$ a first coarse location estimate with an error of $\sim 1.4 \mathrm{~m}$ is available reaching $\sim 0.5 \mathrm{~m}$ location error after $7 \mathrm{~s}$ algorithm working time.

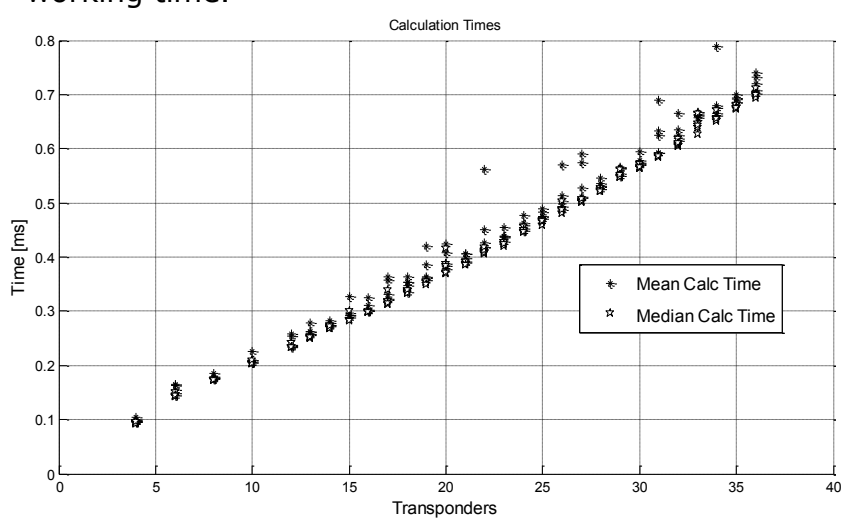

Figure 7. Localization algorithm computation time

Figure 8 illustrates the localization error over the measurement time. As you can see in the graph the original approach needs approximately $7.3 \mathrm{~s}$ to calculate a first result. Within this time the clustering approach can provide $\sim 25$ estimates with a result rate of $\sim 3$ estimates per second. By adjusting the clustering steps the time for the next calculation step and the precision degree could be fitted dynamically.

\section{Conclusion}

In this paper we propose a clustering algorithm for a device-free passive RFID based localization approach. It compensates an inherent system tradeoff between measurement speed and localization precision.

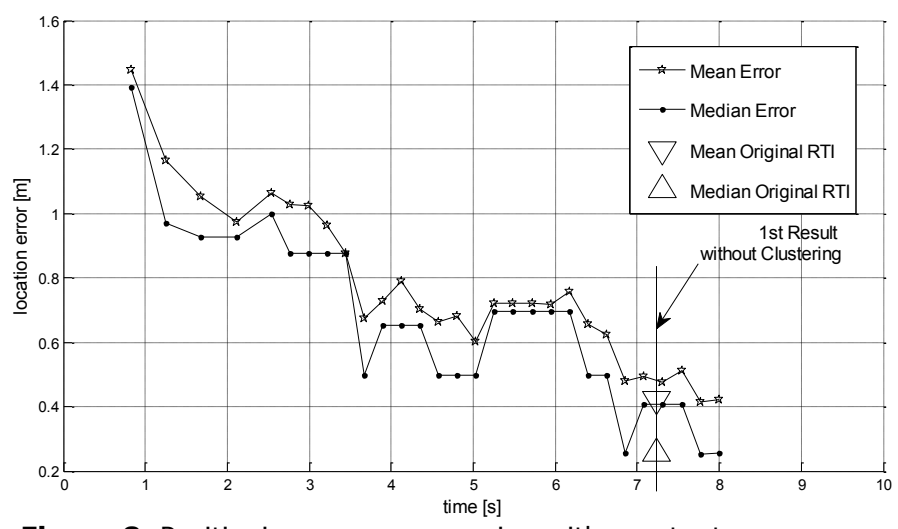

Figure 8. Positioning error progression with constant clustering rate

The clustering approach divides the calculation process in to a first coarse estimation part which is very fast and provides a first idea of the user's position within a larger confidence area. Giving that results into the second clustering phase the distinct communication with sensor subsets provides an adjustable localization process with decreasing positioning errors over time. In our validation we reach a location error of $\sim 1.4 \mathrm{~m}$ after $0.8 \mathrm{~s}$ reaching $\sim 0.4 \mathrm{~m}$ after $7 \mathrm{~s}$ with a result rate of $\sim 3$ results per second. By comparison: the original approach reaches its first estimate with an equal location error not until $\sim 7.2 \mathrm{~s}$.

Enhancing the speed of the approach leads to multiple advantages for superimposed intention recognition system within smart environments, i.e. starting intention guesses were symbolic localization results or areas are sufficient.

The available RFID communication protocols are the bottleneck of every superimposed algorithm or 
approach which is not using the technology in the common practice. Therefore we will have to work on a new adjusted protocol to get rid of the mentioned measurement related disadvantages.

\section{Acknowledgements}

We want to thank Björn Striebing for his supporting experimental work. This work was partially financed by the German Research Foundation (DFG) within the graduate school Multimodal Smart Appliance ensembles for Mobile Applications (MuSAMA, GRK 1424).

\section{References}

[1] D. Lieckfeldt, J. You, and D. Timmermann, "Characterizing the Influence of Human Presence on Bistatic Passive RFID-System," 2009 IEEE International Conference on Wireless and Mobile Computing, Networking and Communications, pp. 338-343, Oct. 2009.

[2] B. Wagner, B. Striebing, and D. Timmermann, "A System for Live Localization In Smart Environments," IEEE International Conference on Networking, Sensing and Control, 2013.

[3] D. Lieckfeldt, J. You, and D. Timmermann, "Exploiting RF-Scatter: Human Localization with Bistatic Passive UHF RFID-Systems," 2009 IEEE International Conference on Wireless and Mobile Computing, Networking and Communications, no. Ic, pp. 179-184, Oct. 2009.

[4] B. Wagner and N. Patwari, "Passive RFID Tomographic Imaging for Device-Free User Localization," Workshop of Positioning, Navigation and Communication, no. 1, pp. 1-6, 2012.

[5] J. Skodzik, V. Altmann, and B. Wagner, "A Highly Integrable FPGA-Based Runtime-Configurable Multilayer Perceptron," 27th IEEE International Conference on Advanced Information Networking and Applications (AINA-2013), 2013.
[6] B. Wagner, D. Timmermann, G. Ruscher, and T. Kirste, "Device-free User Localization utilizing Neural Networks and passive RFID," Conference on Ubiquitous Positioning Indoor Navigation and Location Based Service (UPINLBS), 2012.

[7] J. Wilson and N. Patwari, "Through-Wall Motion Tracking Using Variance-Based Radio Tomography Networks," arXiv. org, Oct, pp. 1-9, 2009.

[8] J. Wilson and N. Patwari, "Radio Tomographic Imaging with Wireless Networks," IEEE Transactions on Mobile Computing, vol. 9, no. 5, pp. 621-632, May 2010.

[9] U. Ahmad, A. Gavrilov, and U. Nasir, "In-building localization using neural networks," Intelligent Systems, IEEE, 2006.

[10] S. Ergut, R. R. Rao, O. Dural, and Z. Sahinoglu, "Localization via TDOA in a UWB Sensor Network using Neural Networks," 2008 IEEE International Conference on Communications, pp. 2398-2403, 2008.

[11] A. Shareef, Y. Zhu, and M. Musavi, "Localization Using Neural Networks in Wireless Sensor Networks," Proceedings of the 1st International ICST Conference on Mobile Wireless Middleware, Operating Systems and Applications, 2008.

[12] EPCGlobal Inc., "Specification for RFID Air Interface EPC ${ }^{\mathrm{TM}}$ Radio-Frequency Identity Protocols Class-1 Generation-2 UHF RFID Protocol for Communications at $860 \mathrm{MHz}-960 \mathrm{MHz}$," no. October, 2008.

[13] "Alien Technology Inc." [Online]. Available: http://www.alientechnology.com/. 